\title{
Fiber optic thermal detection of composite delaminations
}

\author{
Meng-Chou $\mathrm{Wu}^{*}$ and William P. Winfree \\ NASA, Langley Research Center, MS 231, Hampton, VA, USA 23681-2199
}

\begin{abstract}
A recently developed technique is presented for thermographic detection of delaminations in composites by performing temperature measurements with fiber optic Bragg gratings. A single optical fiber with multiple Bragg gratings employed as surface temperature sensors was bonded to the surface of a composite with subsurface defects. The investigated structure was a 10-ply composite specimen with prefabricated delaminations of various sizes and depths. Both during and following the application of a thermal heat flux to the surface, the individual Bragg grating sensors measured the temporal and spatial temperature variations. The data obtained from grating sensors were analyzed with thermal modeling techniques of conventional thermography to reveal particular characteristics of the interested areas. Results were compared and found to be consistent with the calculations using numerical simulation techniques. Also discussed are methods including various heating sources and patterns, and their limitations for performing in-situ structural health monitoring.
\end{abstract}

Keywords: fiber Bragg grating, temperature sensor, thermal health monitoring, thermography, composite, delamination

\section{INTRODUCTION}

Fiber optic sensors have been extensively studied and proposed for temperature and strain sensing in heath monitoring systems of aerospace structures and materials as well as many other applications ${ }^{1,2}$ Compared to other sensors, fiber optic sensors have the advantages of being lightweight and flexible, and requiring simpler wiring especially for distributed sensing. For an extensive heath monitoring system distributed sensors are an important requirement. Most efforts have focused on using distributed fiber optic strain sensing systems, with limited consideration given to temperature sensing systems. For fiber optic temperature sensing, the conventional techniques using Raman scattering can detect the average temperature over a long distance within a single fiber. ${ }^{3}$ However the spatial resolutions for temperature readings-averaging over a length of fiber-are typically about one meter. Therefore they are not suitable for measuring the thermal responses at specific locations of the investigated materials.

Another more attractive alternative is using fiber Bragg gratings (FBGs) for distributed temperature sensing (DTS). FBGs written in an optical fiber have a physical length of only a few millimeters. This size makes the FBG nearly a point sensor. The thermal response of FBGs is relatively linear in the range around room temperature, though they have only moderate temperature sensitivities. Nevertheless, if the sensing fiber is bonded to a substrate, which is the investigated material, the temperature sensitivity can be significantly enhanced by the thermal expansion coefficient of the material for most cases. There are two types of DTS systems. One uses multiple FBGs written at different (multiplexing) wavelengths and read with a time domain demodulation system. However the total number of these FBGs in a singe fiber is limited due to the finite bandwidth of the laser in the detector system. ${ }^{1}$ The other one uses low reflectivity FBGs written at the same nominal wavelength and read with a frequency domain demodulation system., ${ }^{4,5}$ This technique allows a single fiber to contain hundreds of FBGs, potentially employed as temperature sensors.

An effective fiber optic DTS system has some advantages compared to a distributed strain sensing system for structure health monitoring. Strain sensing systems require mechanical excitation of the investigated structure or material. ${ }^{6}$ For some cases this is impractical, if not impossible. Obviously, employing a DTS system requires the investigated material to be thermally excited. However, the thermal excitation needs only to produce temperature changes ranging from a few

*Meng-Chou.Wu-1@nasa.gov; phone 1757864 4951; fax 17578644914 
degrees to tens of degrees. Such temperature variation is comparable with the excited temperature changes for conventional thermography techniques.

Conventional thermographic techniques, in general, utilize a flash or quartz lamp as a heating source and an infrared imager to detect the thermal response of the investigated material. The heating pulse duration typically ranges from a fraction of a second to a few seconds. The IR imager contains an array of several hundred by several hundred detectors. These thermographic techniques are capable of large area inspection of aerospace structures and materials for their reliability and safety. One of the particularly important areas is the inspection of graphite fiber reinforced composite materials because they are being increasingly used as primary structures due to their high stiffness and strength to weight ratio. Of particular interest is the detection of delaminations that can appreciably reduce the compressive strength of a composite. Recently Winfree et al. successfully developed a physical model of two-layered systems for accurate reduction of the temporal thermal response of a composite with fabricated delaminations to the depths of the delaminations. ${ }^{7}$

In this paper we propose a new technique using a single optical fiber with multiple FBGs for the thermographic detection of flaws in materials and structures. The investigated structure was a 10-ply composite specimen with fabricated delaminations of various sizes and depths, similar to the one mentioned above. The optical fiber was bonded to the surface of the investigated composite. Both during and following the application of a thermal heat flux to the surface, the individual Bragg grating sensors measured the temporal and spatial temperature variations. The data obtained from individual FBGs were analyzed with thermal modeling to reveal particular characteristics within the area of interest.

\section{THEORY}

\subsection{FBG as a temperature sensor}

In general, a fiber Bragg grating can be characterized by its Bragg wavelength, which is the center wavelength of light reflected from the grating. The Bragg wavelength is given as

$$
\lambda_{\mathrm{B}}=2 \mathrm{n}_{\mathrm{eff}} \Lambda
$$

where $n_{\text {eff }}$ is the effective refractive index of the fiber core and $\Lambda$ the grating period. For a fiber Bragg grating bonded onto or embedded in a polymeric substrate, a change in the temperature causes a change in the grating period due to not only the thermal expansion of the fiber but also the strain induced by thermal expansion of the substrate. In addition, the refractive index of the fiber core changes because of the thermo-optic effect. Combining all the above effects, the shift in Bragg wavelength due to a temperature change, $\Delta T$, is given as

$$
\delta \lambda_{\mathrm{B}} / \lambda_{\mathrm{B}}=\left(1-\mathrm{p}_{\mathrm{e}}\right) \delta l / l+\delta \mathrm{n}_{\mathrm{eff}} / \mathrm{n}_{\mathrm{eff}},
$$

where $\mathrm{p}_{\mathrm{e}}$ is the photoelastic constant of the optical fiber and $\delta l / l$ is the combined thermally induced strain of the fiber. In general the photoelastic constant, the fractional index change, and the thermal expansion coefficients of the fiber and the substrate are temperature dependent and nonlinear, especially at low temperatures. However, for a finite temperature change, especially for the temperatures around and above the room temperature, Eq. (2) can be rewritten as a linear form as, ${ }^{8}$

$$
\delta \lambda_{\mathrm{B}} / \lambda_{\mathrm{B}}=\left[\left(1-\mathrm{p}_{\mathrm{e}}\right) \alpha_{\mathrm{p}}+\xi\right] \Delta T
$$

where $\xi=\left(1 / \mathrm{n}_{\text {eff }}\right)\left(\partial \mathrm{n}_{\mathrm{eff}} / \partial T\right)$, is the thermo-optic coefficient of the fiber and $\alpha_{\mathrm{p}}$ is the thermal expansion coefficient of the substrate. Eq. (3) takes into account the conditions when the thermal expansion coefficient and the physical dimension of the substrate are much greater than those of the optical fiber. This simple equation allows the FBG to perform as a temperature sensor of the substrate.

\subsection{Simulation of thermal response of composite with delamination}

A one-dimensional representation has previously been shown to accurately model the thermal response of a composite with a delamination that is significantly larger than the thickness of the composite. ${ }^{7}$ In this study, a fiber optic sensor is 
between a piece of Kapton ${ }^{\circledR}$ tape and the surface of the composite. A composite with a delamination and Kapton ${ }^{\circledR}$ tape on the surface is represented as the three-layer system as shown in Fig. 1. The heated side of the composite has the Kapton ${ }^{\circledR}$ tape and fiber optic sensor (not shown). The composite is represented as two layers with a contact resistance between the two layers. The contact resistance $(R)$ is the thickness of the delamination air gap divided by the thermal conductivity of air. The two composite layers are assumed to have the same thermal conductivity and diffusivity, but different thicknesses.

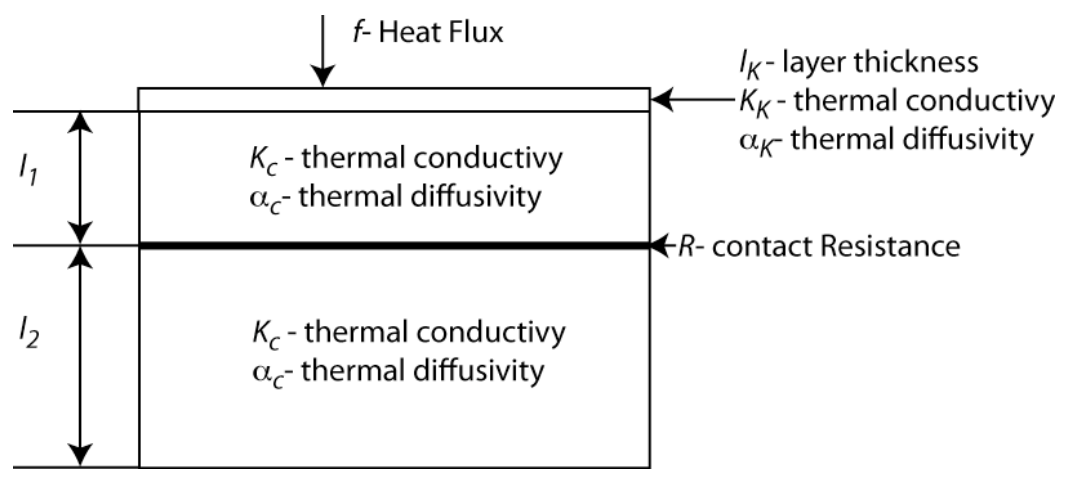

Fig 1. One dimension model of Kapton ${ }^{\circledR}$ tape on composite with delamination. The delamination is represented as a contact resistance between two layers of different thicknesses, but with the same thermal properties.

The Laplace transform of the front surface temperature $\left(v_{0}(s)\right)$ and flux $(f(s))$ can be related to the temperature at the back surface $\left(v_{l}(s)\right)$ by a series of transfer matrices:

$$
\left[\begin{array}{cc}
A_{c} & B_{c} \\
C_{c} & D_{c}
\end{array}\right]\left[\begin{array}{cc}
\cosh \left(l_{K} p_{K}\right) & -\sinh \left(l_{K} p_{K}\right) / K_{K} p_{K} \\
-K_{K} p_{K} \sinh \left(l_{K} p_{K}\right) & \cosh \left(l_{K} p_{K}\right)
\end{array}\right]\left[\begin{array}{c}
v_{0}(s) \\
f(s)
\end{array}\right]=\left[\begin{array}{c}
v_{1}(s) \\
0
\end{array}\right]
$$

where $s$ is the Laplace variable, $K_{K}$ is the Kapton ${ }^{\circledR}$ thermal conductivity, $p_{K}=\left(s / \alpha_{K}\right)^{1 / 2}$ and $\alpha_{K}$ is the thermal diffusivity of the Kapton ${ }^{\circledR}$. The left matrix represents the transfer matrix associated with the composite,

$$
\left[\begin{array}{ll}
A_{c} & B_{c} \\
C_{c} & D_{c}
\end{array}\right]=\left[\begin{array}{cc}
\cosh \left(l_{2} p_{c}\right) & -\sinh \left(l_{2} p_{c}\right) / K_{c} p_{c} \\
-K_{c} p_{c} \sinh \left(l_{2} p_{c}\right) & \cosh \left(l_{2} p_{c}\right)
\end{array}\right]\left[\begin{array}{cc}
1 & -R \\
0 & 1
\end{array}\right]\left[\begin{array}{cc}
\cosh \left(l_{1} p_{c}\right) & -\sinh \left(l_{1} p_{c}\right) / K_{c} p_{c} \\
-K_{c} p \sinh \left(l_{1} p_{c}\right) & \cosh \left(l_{1} p_{c}\right)
\end{array}\right],
$$

where $l_{l}$ is the depth of the delamination, $l_{2}$ is the thickness of the composite minus $l_{l}, K_{c}$ is the composite thermal conductivity, $p_{c}=\left(s / \alpha_{c}\right)^{1 / 2}$ and $\alpha_{c}$ is the thermal diffusivity of the composite.

Eq. (4) is solved for the Laplace transform of the front surface temperature $\left(v_{0}(s)\right)$. The Laplace transform of temperature at the fiber sensor is calculated using the first layer transfer matrix to be

$$
v_{f}(s)=v_{0}(s) \cosh \left(l_{K} p_{K}\right)-\frac{f(s) \sinh \left(l_{K} p_{K}\right)}{K_{K} p_{K}}
$$

The time domain impulse response, $v_{f}(t)$ can be found by setting $f(s)$ to 1 and performing a numerical inversion of Eq. (6) using Talbot's method.

The time response for quartz heating is found by convolving the impulse response with the flux from the quartz lamps $(F(t))$. The flux from a quartz lamp heat source is assumed to have the temporal profile shown in Fig. 2. As shown in the 
figure, after the lamps are turned on, the flux is assumed to linearly increase to a maximum flux $\left(F_{\mathrm{m}}\right)$ in time $t_{l}$. The flux is then assumed to be constant from $t_{1}$ to $t_{2}$, and then linearly decrease from $t_{2}$ to $t_{3}$. At $t_{4}$, the heating cycle begins again. $t_{4}$ and $t_{2}$ correspond to the setting of the function generator turning on and off the quartz lamps. $t_{1}$ and $t_{3}-t_{2}$ are the times required for the quartz lamps to come to full power and cool respectively.

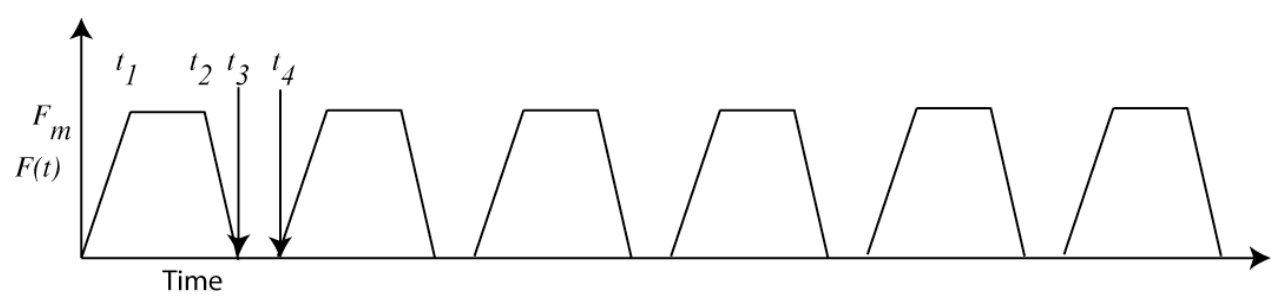

Fig. 2. Assumed shape of the heat flux from quartz lamp.

The thermal response of the fiber at the back surface of Kapton ${ }^{\circledR}$ tape on the composite is found by convolving the impulse response with the flux from the quartz lamps and is given by

$$
v_{c}(t)=\int_{0}^{t} F(\tau) v_{f}(t-\tau) d \tau
$$

The convolution could be performed before the numerical inversion, however experience indicates the numerical inversion is more stable. Therefore to determine the thermal response for the periodic heat source, the inverse Laplace transform of the periodic excitation is found numerically and convolved with the impulse response.

\section{EXPERIMENT}

The low (smaller than a few tenths of one percent) reflectivity FBGs used in this research were written in situ, into the optical fiber drawn by using the NASA Langley optical fiber draw tower. They were written with a pulsed KrF-excimer laser of $248 \mathrm{~nm}$ and a Talbot interferometer arrangement mounted on the tower. The interferometer consisted of a phase mask functioning as a beam splitter and a pair of mirrors used to recombine the split beams to form an interference pattern. An aperture was placed in the laser beam path to control the grating length and spatial profile. ${ }^{9}$ FBG lengths for the present study were nominally $5 \mathrm{~mm}$. The grating pitch written into the fiber could be adjusted by changing the relative angle of the two mirrors. These single-mode fibers were drawn from commercially available germanium-doped preforms of high numerical apertures. The drawn fibers with FBGs were coated with polyimide to thicknesses ranging from 11 to 16 micrometers, and then ink-marked to show grating locations.

These low reflectivity FBGs were interrogated using a frequency domain demodulation system shown in Fig. 3 . In this system, the fiber coupler $\mathrm{C}_{1}$ and a pair of Faraday rotation mirrors (FRMs) form an in-fiber interferometer with an optical path difference of $2 \mathrm{n}_{\mathrm{eff}} \mathrm{L}_{0}$, where $\mathrm{n}_{\mathrm{eff}}$ is the effective refractive index of the fiber core and $\mathrm{L}_{0}$ the length of the reference cavity. The signals are driven by the tuning of the laser and detected at the photo-detector $\mathrm{D}_{1}$. They are used to trigger the sampling of signal at $\mathrm{D}_{2}$, which is the output of another in-fiber interferometer formed with the fiber coupler $\mathrm{C}_{2}$, a broadband reflector, and a particular fiber Bragg grating at a distance of $\mathrm{L}_{\mathrm{i}}$. If there is a series of low reflectivity Bragg gratings written at the same wavelength on a single fiber at different locations, the reflected signals from each grating are superimposed and detected at $\mathrm{D}_{2}$. The detected signals are further processed to obtain the spatial spectrum of all gratings, which displays the physical profiles of the gratings at different locations. The spatial spectrum of a particular grating can then be windowed for investigating individual gratings. Fig. 4 shows the spatial spectrum of two FBGs in a fiber with multiple gratings. The gratings have a physical length of about $5 \mathrm{~mm}$ and a separation distance of $10 \mathrm{~cm}$. The signals from the demodulation system are further processed for the strain (thermally induced strain, in this case) of each point within the physical length of a grating. Averaging over a certain number of points within the grating length is taken for the strain of the particular grating. 


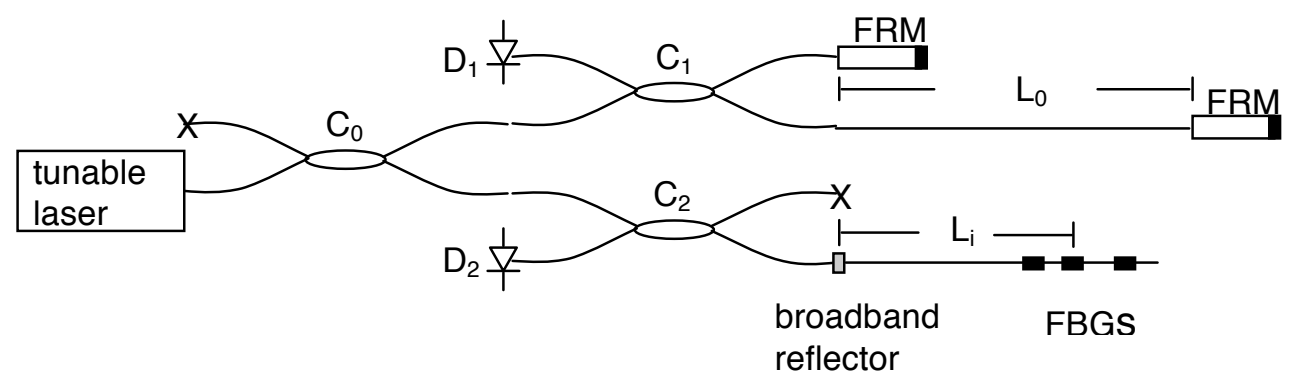

Fig. 3. Schematic diagram of a frequency domain demodulation system. Items $C_{0}, C_{1}$, and $C_{2}$ are fiber couplers. " $X$ " indicates that the unused port is terminated. Items $\mathrm{D}_{1}$ and $\mathrm{D}_{2}$ are detectors and FRMs are Faraday rotation mirrors.

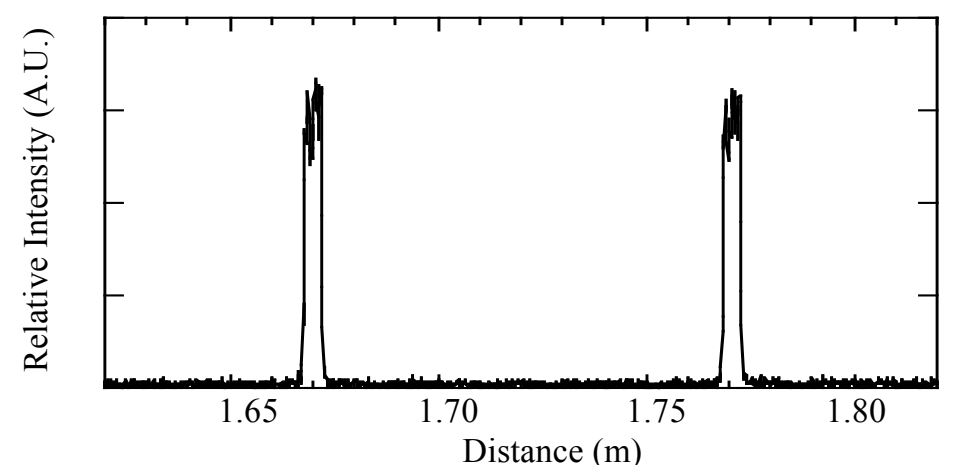

Fig. 4. The spatial spectrum of two FBGs with a physical length of about $5 \mathrm{~mm}$ and a separation distance of $10 \mathrm{~cm}$.

In this study, an optical fiber with 32 FBGs was bonded to an investigated composite specimen with Kapton ${ }^{\circledR}$ tape, as shown in Fig 5 (a). This bonding technique allowed the optical fiber to be bonded and taken off the sample quickly and neatly. At the same time it also allowed the fiber to sense the thermal strain for a temperature change up to $50^{\circ} \mathrm{C}$ without imminent slipping.

The specimen used for testing the viability of this measurement technique was a composite panel with 20 delaminations at specified depths. The 10-ply quasi-isotropic composite panel with a lay-up of $[0,45,90,-45,0 /, 45,90,-45 /, 0,90 /]$ was $31.75 \times 31.75$ centimeters and 0.19 centimeters thick (Fig. 5(a)). The delamination defect areas were squares with sizes of $3.8 \times 3.8,2.5 \times 2.5,1.9 \times 1.9$, and $1.3 \times 1.3$ square centimeters. The defects were buried at depths of $10,20,30,40$, and 50 percent of the total thickness. A schematic of the defect layout and FBG sensor numbers is shown in Fig. 5 (b). The optical fiber bonded to the specimen had FBGs with a separation (center-to-center) distance of 5.08 centimeters, which was the same as the separation distance of the squares in the same row. One FBG was placed at the center of each square. Five FBGs numbered 14-19 were outside the squares for comparison.

The experimental setup is shown in Fig. 6. A quartz lamp was used to heat the front and the back surface of the specimen with a single or multiple heating cycles. The cyclic heating controlled by a function generator had a 2 second period with a $50 \%$ duty-cycle, and a total duration of about 20 seconds. The grating data acquisition was performed during and following the application of the heat flux to the surface at a rate of $100 \mathrm{~Hz}$. Ideally, it is desirable to acquire data from all of the fiber optic sensors at one time. At the higher acquisition rate used for these measurements $(100 \mathrm{~Hz})$ this was not possible. Instead it was only possible to collect data along 5 adjacent gratings. However for each set, the timing of 
the data acquisition and the application of the heat flux (the trigger of the function generator) were synchronized as best as possible.

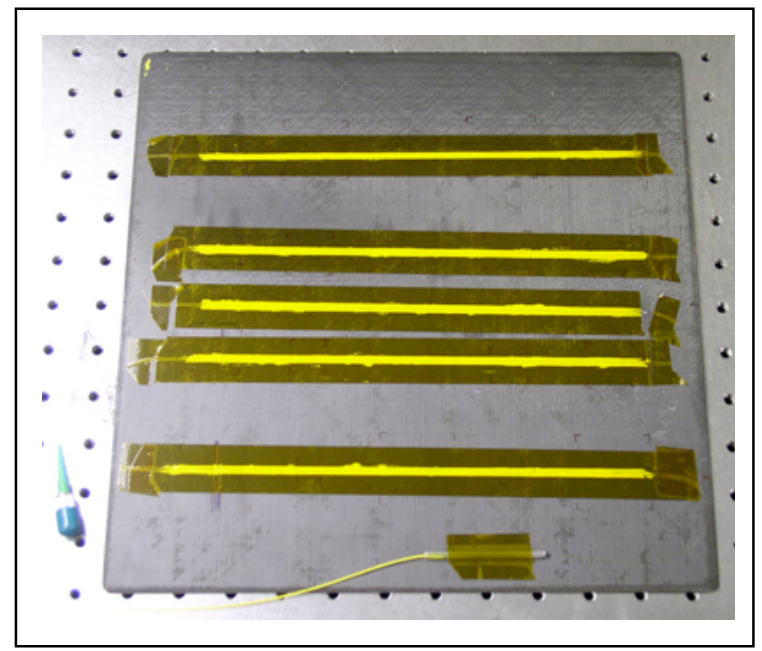

(a)

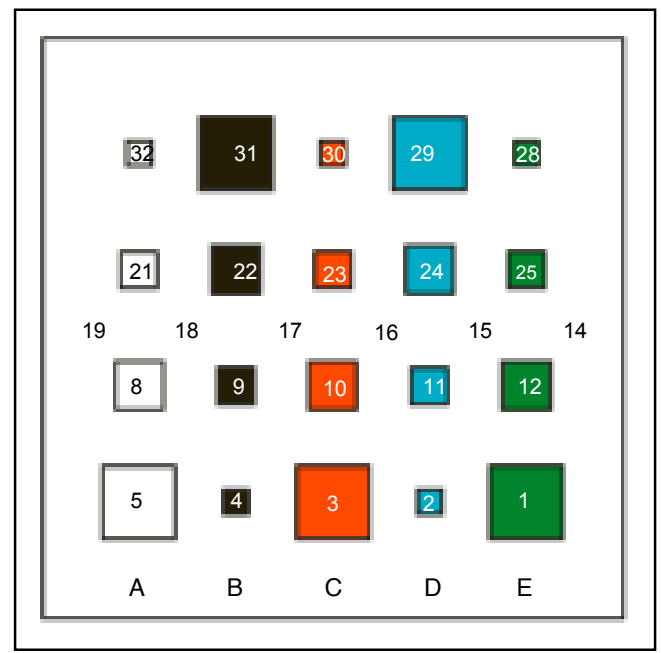

(b)

Fig. 5. (a) A single optical fiber with multiple Bragg grating sensors bonded onto the surface of a 10-ply-composite. (b) The fabricated delaminations of various sizes arranged in columns between plies 1-2 (A), 2-3 (B), 3-4 (C), 4-5 (D), 5-6 (E).

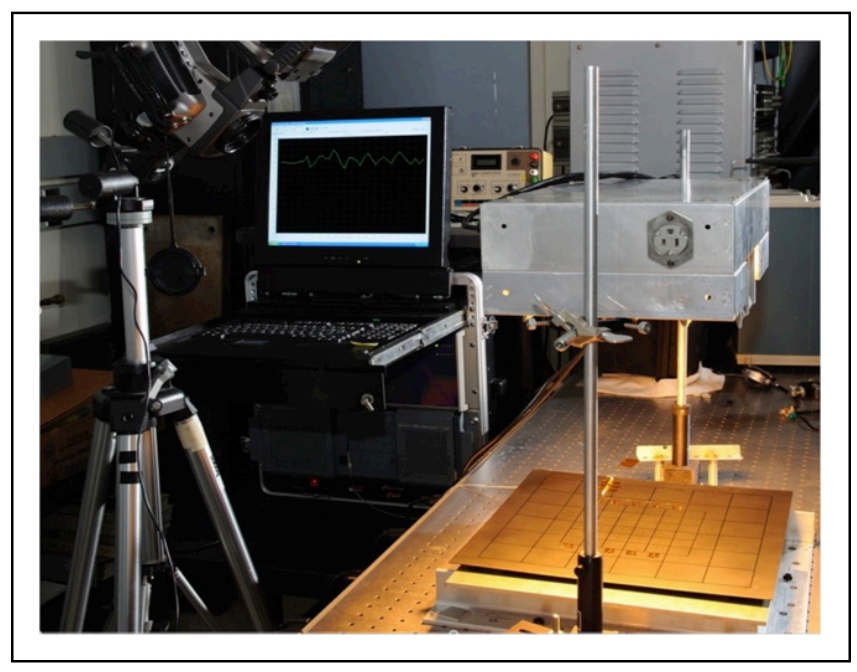

Fig. 6. The experimental setup of the fiber optic thermal health monitoring system with a quartz lamp as the heating source.

\section{RESULTS AND DISCUSSION}

Simulations were performed for a $0.19 \mathrm{~cm}$ thick composite with $0.00254 \mathrm{~cm}$ of Kapton ${ }^{\circledR}$ on the surface, as well as simulations for delamination with depths of $0.019 \mathrm{~cm}, 0.057 \mathrm{~cm}$, and $0.095 \mathrm{~cm}$ below the heated surface. These simulations results are shown in Fig. 7. The simulations were performed assuming the thermal diffusivity of the composite was $0.0051 \mathrm{~cm}^{2} / \mathrm{sec}$ based on through transmission measurements and a thermal conductivity of 97000 $\mathrm{Erg} /(\mathrm{cm} \mathrm{K} \mathrm{sec})$ and a delamination air gap separation of $0.01 \mathrm{~cm}$. The thermal properties for Kapton ${ }^{\circledR}$ were values from 
"Summary of Properties of Kapton ${ }^{\circledR}$ Polyimide" published by DuPont ${ }^{\mathrm{TM}}{ }^{10}$. The flux parameters were $t_{1}=0.35$ sec, $t_{2}=$ $1.024 \mathrm{sec}, t_{3}=1.324 \mathrm{sec}$ and $t_{4}=2.048 \mathrm{sec}$, chosen to correspond to the experimental values.

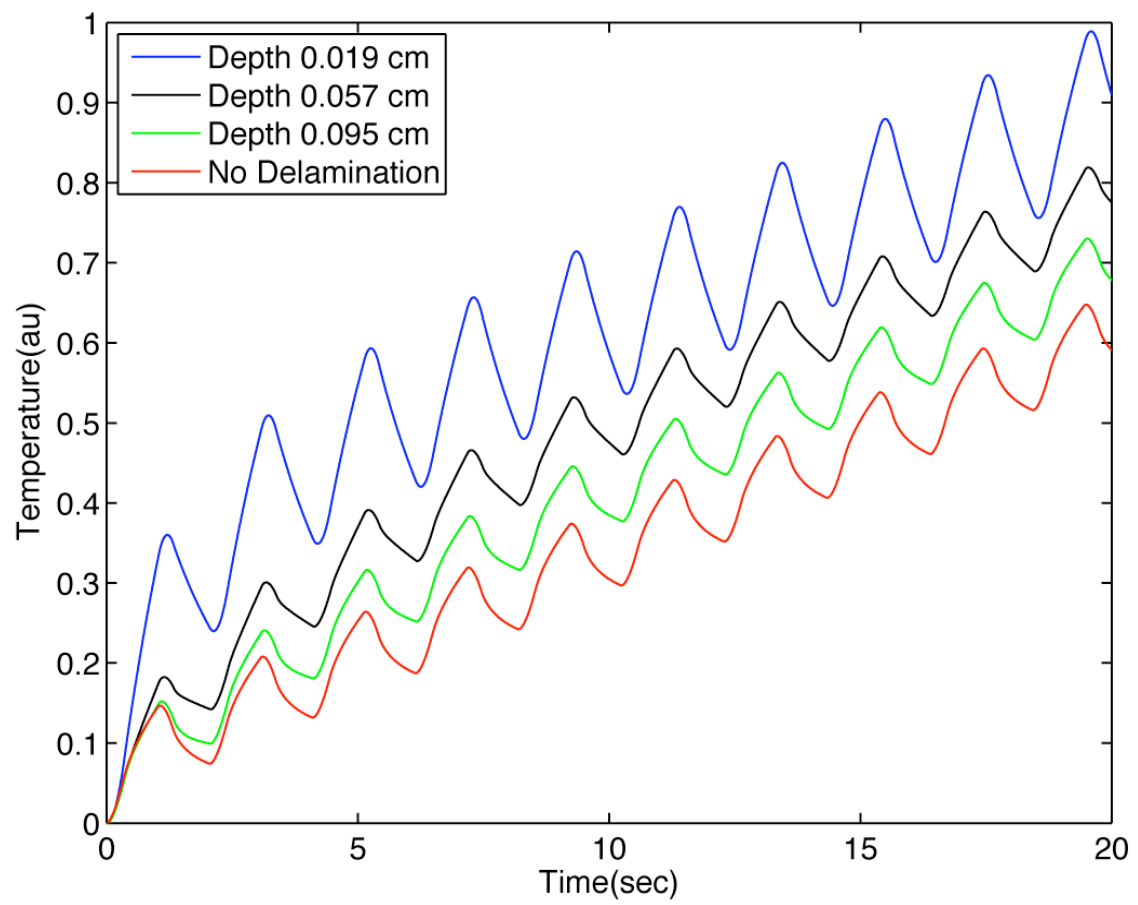

Fig. 7. Simulation results for temperature measurement at the back surface of Kapton ${ }^{\circledR}$ tape on the composite with delaminations at different depths from the heated surface.

As can be seen from the simulation shown in Fig. 7, there is a significant difference between the shape of the thermal response of the delamination closest to the surface and the rest of the responses. The deepest delamination simulated has a thermal response shape that is close to the shape of no delamination. The finite contact resistance of the delamination results in the most significant temperature variations per cycle for the delamination closest to the surface. If the contact resistance was infinity (effectively no back layer), simulations indicate that for the delamination closest to the surface, during the portion of the cycle with no heating, the temperature is approximately constant.

The data acquired from the fiber optic measurements were analyzed using the model developed in section 2.2. Proper determination of the lamp characteristics $\left(t_{1}, t_{2}, t_{3}\right.$ and $\left.t_{4}\right)$ was required for accurate comparison. $t_{2}$ and $t_{4}$ are fixed by the function generator that turns on and off the flash lamps. $t_{1}$ and $t_{3}$ were determined by fitting all of the data sets and requiring all of the $t_{1}$ and $t_{3}$ to be the same for all measurement. Best results were found by fitting only the second half of the data as shown in Fig. 8. This may in part be a result of the $t_{l}$ and $t_{3}$ changing until the lamps warm up.

The last 10 seconds of the time records were fit, varying the depth and contact resistance of delamination. The depth of the delamination estimated from a fit of the data using the model for different depths and sizes of delamination is shown in Fig 9. The absolute thicknesses were determined from the thickness of the plies and the placement of the delaminations. As can be seen from the figure, the fits give an accurate characterization of the depth for thicknesses less than or equal to $0.076 \mathrm{~cm}$ deep (the first four groups), with the average difference between estimated depth and absolute depth ranging from $0.003 \mathrm{~cm}$ to $-0.006 \mathrm{~cm}$, less than $15 \%$ of the actual depth. The estimated depth of the deepest delamination between the fifth and sixth plies $(0.095 \mathrm{~cm}$ below surface) has a large significant error, $-0.02 \mathrm{~cm}$ (approximately 1 ply thickness). The fact that the largest error occurs for the largest area delamination at the deepest depth indicates the systematic error is not due to the one-dimensional model becoming an inappropriate method for analyzing the data. 


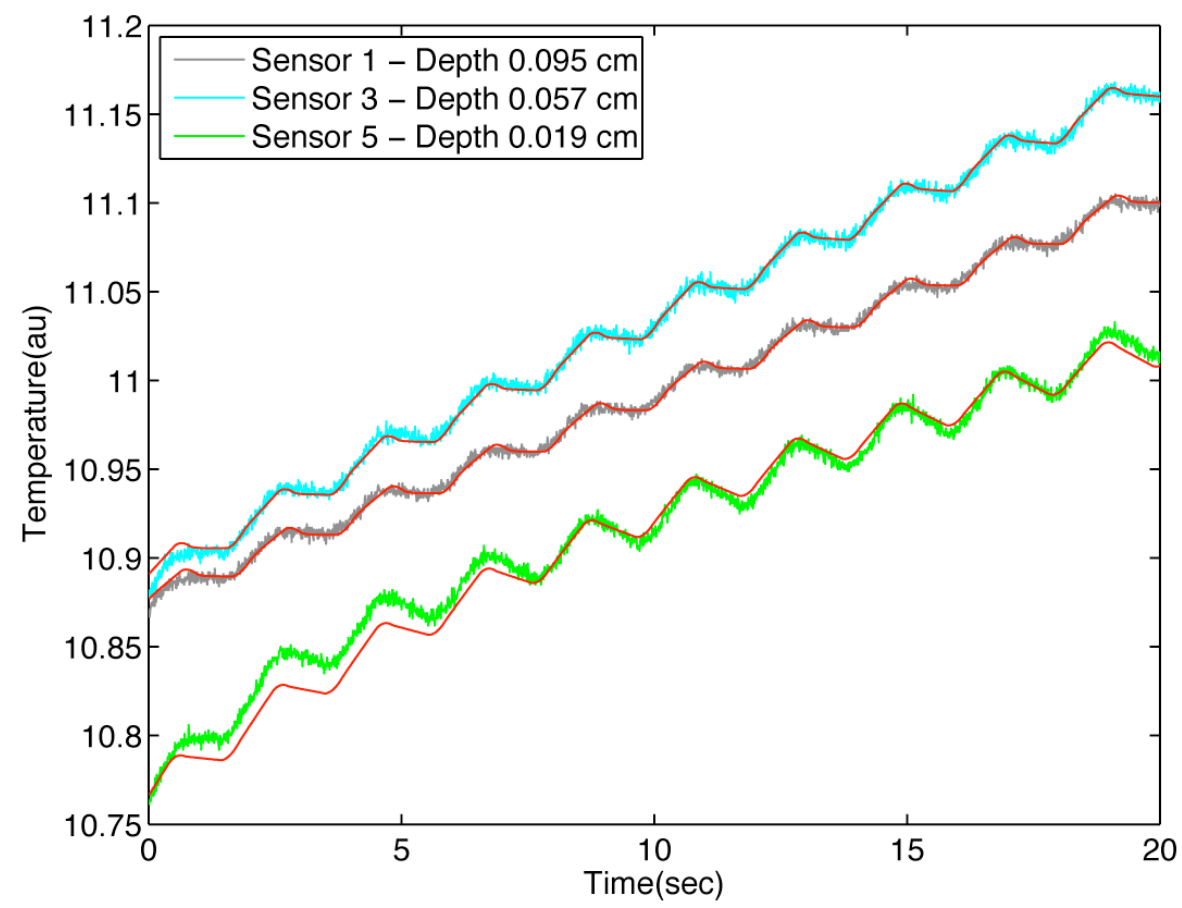

Fig. 8. Results of the fit of data from fiber optic sensors using the one-dimensional model discussed in section 2.2.

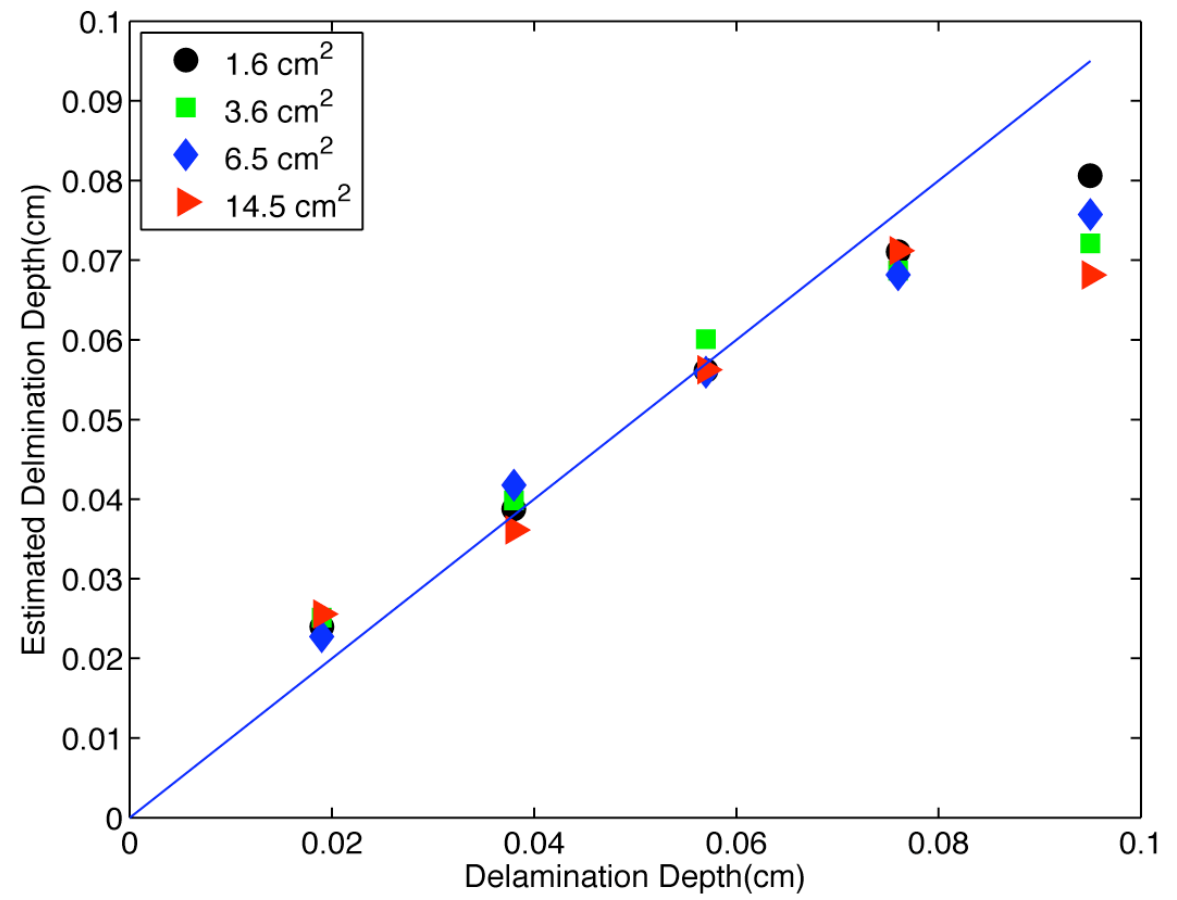

Fig. 9. The depth of the delamination estimated from a fit of the data using the model for different depths of delamination. The legend indicates the different areas of the delaminations. 


\section{CONCLUSION}

We have developed a new technique using distibuted FBG sensors for thermographic detection of flaws in materials and structures. Individual fibers with multiple FBGs employed as temperature sensors can be quickly bonded to the surfaces of structures with Kapton ${ }^{\circledR}$ tape. By applying a cyclic thermal heat flux to the surface of the investigated structures, the individual Bragg grating sensors successfully measured the temporal and spatial temperature variations on the surface. These thermal responses were consistent with the one-dimensional model of the thermal response for a delaminated composite. The measured depths for delaminations of different depths and sizes are in good agreement with the model predictions. Future efforts will focus on developing the technique with a faster detection system, detecting more FBGs at one time, and assessing its potential for performing thermal health monitoring of aerospace structures and materials.

\section{REFERENCES}

[1] Giles, C. R., "Lightwave applications of fiber Bragg gratings," J. Lightwave Technol. 15, 1391-1404 (1997).

[2] Kersey, A. D., M. A Davis, H. J. Patrick, M. LeBlanc, K. P. Koo, C. G. Askins, M.A. Putnam, E. J. Friebele, "Fiber grating sensors," J. Lightwave Technol. 15, 1442-1463 (1997).

[3] J. P. Dakin, D. J. Pratt, G. W. Bibby, and J. N. Ross, "Distributed optical fiber Raman temperature sensor using a semiconductor light source and detector," Electronics Letters 21, 569-570 (1985).

[4] Wu, M.-C., R. Pater, and S. L. DeHaven, "Effects of coating and diametric load on fiber Bragg gratings as cryogenic temperature sensors," Proc. SPIE, 6933, 693303 (2008).

[5] Froggatt, M. and J. Moore, "Distributed measurement of static strain in an optical fiber with multiple Bragg gratings at nominally equal wavelengths," Appl. Opt. 37, 1741-1746 (1998).

[6] James, S. W., M. L. Dockney, and R. P. Tatam, "Simultaneous independent temperature and strain measurement using in-fiber Bragg grating sensors," Electron. Lett. 32, 1133-1134 (1996).

[7] Winfree, W. P. and J. Zalameda, "Thermographic determination of delamination depth in composites," in Thermosense XXV, Proc. SPIE 5073, 363-373 (2003).

[8] Gupta, S., T. Mizunami, T. Yamao, and T. Shimomura, "Fiber Bragg grating cryogenic temperature sensors," Appl. Opt. 25, 5202-5205 (1996).

[9] Wu, M.-C. and R. S. Rogowski, "Fabrication of self-apodized short-length fiber Bragg gratings," Appl. Opt. 42, 5017-5023 (2003).

[10] "Summary of Properties of Kapton® Polyimide," Copyright (C 2010 DuPont. 